\title{
AUTOMORPHISMS OF COXETER GROUPS AND LUSZTIG'S CONJECTURES FOR HECKE ALGEBRAS WITH UNEQUAL PARAMETERS
}

\author{
CÉDRIC BONNAFÉ
}

\begin{abstract}
Let $(W, S)$ be a Coxeter system, let $G$ be a finite solvable group of automorphisms of $(W, S)$ and let $\varphi$ be a weight function which is invariant under $G$. Let $\varphi_{G}$ denote the weight function on $W^{G}$ obtained by restriction from $\varphi$. The aim of this paper is to compare the a-function, the set of Duflo involutions and the Kazhdan-Lusztig cells associated with $(W, \varphi)$ and to $\left(W^{G}, \varphi_{G}\right)$, provided that Lusztig's Conjectures hold.
\end{abstract}

Let $(W, S)$ be a Coxeter system, with $S$ finite, let $\Gamma$ be a totally ordered abelian group and let $\varphi: W \rightarrow \Gamma$ be a weight function such that $\varphi(s)>0$ for all $s \in S$.

Let $G$ be a group of automorphisms of $W$ stabilizing $S$ and $\varphi$. We denote by $\varphi_{G}$ the restriction of $\varphi$ to the fixed points subgroup $W^{G}$. If $\omega \in S / G$ (the orbit set) is such that $W_{\omega}(=\langle\omega\rangle)$ is finite, we denote by $s_{\omega}$ the longest element of the standard parabolic subgroup $W_{\omega}$ and we set $S_{G}=\left\{s_{\omega} \mid \omega \in S / G\right.$ and $W_{\omega}$ is finite $\}$. Then it is well-known that $\left(W^{G}, S_{G}\right)$ is a Coxeter system and that $\varphi_{G}: W^{G} \rightarrow \Gamma$ is a weight function (such that $\varphi_{G}\left(s_{\omega}\right)>0$ for all $\left.\omega \in S / G\right)$.

With the datum $(W, S, \Gamma, \varphi)$ are associated a Hecke algebra $\mathcal{H}(W, S, \Gamma, \varphi)$ over the ring $\mathbb{Z}[\Gamma]$, a Kazhdan-Lusztig basis $\left(C_{w}\right)_{w \in W}$ of $\mathcal{H}(W, S, \Gamma, \varphi)$, equivalence relations $\sim_{\mathcal{L}}, \sim_{\mathcal{R}}$ and $\sim_{\mathcal{L} \mathcal{R}}$ and two functions a :W $\rightarrow \Gamma$ and $\Delta: W \rightarrow \Gamma$ (see $[\mathrm{L}])$. We set $\mathcal{D}=\{w \in W \mid \mathbf{a}(w)=\Delta(w)\}$. With the datum $\left(W^{G}, S_{G}, \Gamma, \varphi_{G}\right)$, we associate similarly $\sim_{\mathcal{L}}^{G}, \sim_{\mathcal{R}}^{G}, \sim_{\mathcal{L} \mathcal{R}}^{G}, \mathbf{a}_{G}, \Delta_{G}$ and $\mathcal{D}_{G}$. The main result of this paper is the following:

Theorem A. Assume that $G$ is a finite solvable group and that Lusztig's conjectures $\left(P_{1}\right),\left(P_{2}\right),\left(P_{3}\right),\left(P_{4}\right)$ in [L, Chapter 14] hold for the datum $\left(W^{H}, S_{H}, \Gamma, \varphi_{H}\right)$ for all subgroups $H$ of $G$. Let $x, y \in W^{G}$. Then:

\footnotetext{
Received June 9, 2008.

Accepted February 4, 2009.

2000 Mathematics Subject Classification: Primary 20C08; Secondary 20C15.

The author is partly supported by the ANR (Project No. JC07-192339).
} 
(a) $\mathbf{a}_{G}(x)=\mathbf{a}(x)$.

(b) $\mathcal{D}_{G}=\mathcal{D} \cap W^{G}$.

(c) Assume moreover that Lusztig's Conjecture $\left(P_{13}\right)$ in [L, Chapter 14] hold for the datum $\left(W^{H}, S_{H}, \Gamma, \varphi_{H}\right)$ for all subgroups $H$ of $G$. If $? \in\{\mathcal{L}, \mathcal{R}\}$, then $x \sim_{\text {? }} y$ if and only if $x \sim_{?}^{G} y$.

(d) Assume moreover that Lusztig's Conjectures $\left(P_{9}\right)$ and $\left(P_{13}\right)$ in $[\mathrm{L}$, Chapter 14] hold for the datum $\left(W^{H}, S_{H}, \Gamma, \varphi_{H}\right)$ for all subgroups $H$ of $G$. Then $x \sim_{\mathcal{L} \mathcal{R}} y$ if and only if $x \sim_{\mathcal{L} \mathcal{R}}^{G} y$.

Remark. If $G$ is not solvable and if we assume moreover that Lusztig's conjecture $\left(P_{12}\right)$ in [L, Chapter 14] holds, then the statements (a), (b) and (c) of Theorem A hold. It is probable that (d) also holds, but the proof should rely on a really different argument than the one presented here. Indeed, using $\left(P_{12}\right)$ and a theorem of Meinolf Geck $[\mathrm{G}]$, one can reduce the problem to the case where $W_{\omega}$ is finite for all $G$-orbits $\omega$ in $S$. Then, since the automorphism groups of irreducible finite Coxeter systems are solvable, one can assume that $G$ is solvable and apply Theorem A above.

The proof of this Theorem makes essential use of reduction modulo $p$. Indeed, an easy induction argument reduces immediately the problem to the case where $G$ is a $p$-group for some prime number $p$. The main ingredient is then the following: the natural stupid map $\mathcal{H}\left(W^{G}, S_{G}, \Gamma, \varphi_{G}\right) \rightarrow$ $\mathcal{H}(W, S, \Gamma, \varphi)^{G}$ is not a morphism of algebras in general. However, if we denote by $\operatorname{Br}_{G}(\mathcal{H}(W, S, \Gamma, \varphi))$ the quotient of $\mathcal{H}(W, S, \Gamma, \varphi)^{G}$ by the twosided ideal $\sum_{H<G} \operatorname{Tr}_{H}^{G}\left(\mathcal{H}(W, S, \Gamma, \varphi)^{H}\right)$ (Brauer's quotient, see for instance [T, Page 91]), then:

Proposition B. Assume that $G$ is a finite p-group. Then the natural linear map $\mathcal{H}\left(W^{G}, S_{G}, \Gamma, \varphi_{G}\right) \rightarrow \operatorname{Br}_{G}\left(\mathcal{H}(W, S, \Gamma, \varphi)^{G}\right)$ is a morphism of algebras whose kernel is generated by p. Moreover, it preserves the KazhdanLusztig basis.

Acknowledgements. Part of this work was done while the author stayed at the MSRI during the winter 2008. The author wishes to thank the Institute for its hospitality and the organizers of the two programs for their invitation. 


\section{$\S 1$. The set-up}

\section{A. The group $(W, S)$}

Let $(W, S)$ be a Coxeter system (with $S$ finite), let $\ell: W \rightarrow \mathbb{N}$ denote the length function, let $\Gamma$ be a totally ordered abelian group and let $\varphi: W \rightarrow \Gamma$ be a weight function $[\mathrm{L}, \S 3.1]$ that is, a map such that $\varphi\left(w w^{\prime}\right)=\varphi(w)+\varphi\left(w^{\prime}\right)$ whenever $\ell\left(w w^{\prime}\right)=\ell(w)+\ell\left(w^{\prime}\right)$.

Let $A$ be the group algebra $\mathbb{Z}[\Gamma]$ : we will use an exponential notation for $A$, namely $A=\bigoplus_{\gamma \in \Gamma} \mathbb{Z} e^{\gamma}$, where $e^{\gamma} \cdot e^{\gamma^{\prime}}=e^{\gamma+\gamma^{\prime}}$ for all $\gamma, \gamma^{\prime} \in \Gamma$. If $a=\sum_{\gamma \in \Gamma} a_{\gamma} e^{\gamma} \in A$, we denote by $\operatorname{deg} a$ (resp. val $a$ ) the degree (resp. the valuation) of $a$, that is, the element $\gamma$ of $\Gamma$ such that $a_{\gamma} \neq 0$ and which is maximal (resp. minimal) for this condition (by convention, $\operatorname{deg} 0=-\infty$ and val $0=+\infty)$.

We shall denote by $\mathcal{H}$ the Hecke algebra associated with the datum $(W, S, \Gamma, \varphi)$. It is a free $A$-module, with standard basis $\left(T_{w}\right)_{w \in W}$, and the multiplication is entirely determined by the following rules:

$$
\begin{cases}T_{w} T_{w^{\prime}}=T_{w w^{\prime}} & \text { if } \ell\left(w w^{\prime}\right)=\ell(w)+\ell\left(w^{\prime}\right) ; \\ \left(T_{s}-e^{\varphi(s)}\right)\left(T_{s}+e^{-\varphi(s)}\right)=0 & \text { if } s \in S .\end{cases}
$$

Note that this implies that $T_{w}$ is invertible in $\mathcal{H}$ for all $w \in W$. This algebra is endowed with an $A$-anti-linear involution ${ }^{-}: \mathcal{H} \rightarrow \mathcal{H}$ which is determined by the following properties:

$$
\begin{cases}\overline{e^{\gamma}}=e^{-\gamma} & \text { if } \gamma \in \Gamma \\ \bar{T}_{w}=T_{w^{-1}}^{-1} & \text { if } w \in W\end{cases}
$$

By [L, Theorem 5.2], there exists a unique element $C_{w} \in \mathcal{H}$ such that

$$
\left\{\begin{array}{l}
\bar{C}_{w}=C_{w} \\
C_{w} \equiv T_{w} \quad \bmod \mathcal{H}_{<0}
\end{array}\right.
$$

where $\mathcal{H}_{<0}=\bigoplus_{w \in W} A_{<0} T_{w}$, and where $A_{<0}=\bigoplus_{\gamma<0} \mathbb{Z} e^{\gamma}$.

Let $\tau: \mathcal{H} \rightarrow A$ be the unique $A$-linear map such that

$$
\tau\left(T_{w}\right)= \begin{cases}1 & \text { if } w=1 \\ 0 & \text { otherwise }\end{cases}
$$

If $w \in W$, we set

$$
\Delta(w)=-\operatorname{deg} \tau\left(C_{w}\right)
$$


and we denote by $n_{w}$ the coefficient of $e^{-\Delta(w)}$ in $\tau\left(C_{w}\right)$. Finally, if $x, y \in W$, we write

$$
C_{x} C_{y}=\sum_{z \in W} h_{x, y, z} C_{z},
$$

where the $h_{x, y, z}$ 's are in $A$ and satisfy $\overline{h_{x, y, z}}=h_{x, y, z}$.

\section{B. The group $\left(W^{G}, S_{G}\right)$}

Let $G$ be a group of automorphisms of $W$ such that, for all $\sigma \in G$, we have

$$
\sigma(S)=S \quad \text { and } \quad \varphi \circ \sigma=\varphi
$$

If $I$ is a subset of $S$, we denote by $W_{I}$ the (standard parabolic) subgroup of $W$ generated by $I$. If $\omega \in S / G$ is such that $W_{\omega}$ is finite, we denote by $s_{\omega}$ the longest element of $W_{\omega}$. We denote by $S_{G}$ the set of $s_{\omega}$, where $\omega$ runs over the set of $G$-orbits in $S$ such that $W_{\omega}$ is finite. Recall the following proposition [H, Corollary 3.5 and Proof of Proposition 3.4]:

Proposition 1.1. $\left(W^{G}, S_{G}\right)$ is a Coxeter system. Let $\ell_{G}: W^{G} \rightarrow \mathbb{N}$ denote the corresponding length function and let $x, y \in W^{G}$. Then $\ell(x y)=$ $\ell(x)+\ell(y)$ if and only if $\ell_{G}(x y)=\ell_{G}(x)+\ell_{G}(y)$.

Let

$$
\begin{aligned}
\varphi_{G}: W^{G} & \longrightarrow \Gamma \\
w & \longmapsto \varphi(w)
\end{aligned}
$$

denote the restriction of $\varphi$ to $W^{G}$. Then, by Proposition 1.1,

$$
\varphi_{G} \text { is a weight function. }
$$

Therefore, we can define $\mathcal{H}_{G}, \mathcal{H}_{G,<0}, T_{w}^{G}, C_{w}^{G}, \tau_{G}, \Delta_{G}, n_{z}^{G}$ and $h_{x, y, z}^{G}$ with respect to $\left(W^{G}, S_{G}, \Gamma, \varphi_{G}\right)$ in a similar way as $\mathcal{H}, \mathcal{H}_{<0}, T_{w}, C_{w}, \tau, \Delta, n_{z}$ and $h_{x, y, z}$ were defined with respect to $(W, S, \Gamma, \varphi)$.

\section{$\S 2 . \quad$ Brauer quotient}

Hypothesis And notation. From now on, and until the end of Section 3, we fix a prime number $p$ and we assume that $G$ is a finite p-group. 


\section{A. Definition}

For all the facts contained in this subsection, the reader may refer to $[T, \S 11]$ : even though this reference deals only with $\mathcal{O}$-algebras (where $\mathcal{O}$ is a commutative complete local noetherian $\mathbb{Z}_{p}$-algebra) which are $\mathcal{O}$-modules of finite type, the proofs can be applied almost word by word to our slightly more general situation.

Let $R$ be a commutative ring and let $M$ be an $R G$-module. If $H$ is a subgroup of $G$, we set

$$
\begin{aligned}
\operatorname{Tr}_{H}^{G}: M^{H} & \longrightarrow \sum_{\sigma \in[G / H]} M^{G} \sigma(m) . \\
m & \longmapsto
\end{aligned}
$$

Here, $[G / H]$ denotes a set of representatives classes in $G / H$. We also define

$$
\operatorname{Tr}(M)=\sum_{H<G} \operatorname{Tr}_{H}^{G}\left(M^{H}\right)
$$

This is an $R$-submodule of $M^{G}$, containing $p M^{G}$. The Brauer quotient $\operatorname{Br}_{G}(M)$ is then defined by

$$
\operatorname{Br}_{G}(M)=M^{G} / \operatorname{Tr}(M)
$$

and we denote by $\operatorname{br}_{G}: M^{G} \rightarrow \operatorname{Br}_{G}(M)$ the canonical map.

Lemma 2.1. Assume that $p R \neq R$ and that $M$ admits an $R$-basis $\mathcal{B}$ which is permuted by the action of $G$. Then $\operatorname{Br}_{G}(M)$ is a free $R / p R$-module with basis $\left(\operatorname{br}_{G}(b)\right)_{b \in \mathcal{B}^{G}}$.

If $M$ is an $R$-algebra and if $G$ acts on $M$ by automorphisms of algebra, then $\operatorname{Tr}(M)$ is a two-sided ideal of $M^{G}$ and so $\operatorname{Br}_{G}(M)$ is an $R$-algebra. Of course, $b_{G}$ is a morphism of algebras in this case. We recall the following result:

Lemma 2.2. Assume that $p R \neq R$, that $M$ is an $R$-algebra, that $G$ acts on $M$ by automorphisms of algebra, that $M$ admits an $R$-basis $\mathcal{B}$ which is permuted by $G$ and let us write $a b=\sum_{c \in \mathcal{B}} \lambda_{a, b, c}$ for $a, b \in \mathcal{B}$. If $a, b \in \mathcal{B}^{G}$, then

$$
\operatorname{br}_{G}(a) \operatorname{br}_{G}(b)=\sum_{c \in \mathcal{B}^{G}} \pi\left(\lambda_{a, b, c}\right) \operatorname{br}_{G}(c),
$$

where $\pi: R \rightarrow R / p R$ is the canonical morphism. 


\section{B. Applications to Hecke algebras}

Since $G$ stabilizes $S$ and $\varphi$, it also acts on $\mathcal{H}$ by automorphisms of $A$-algebra (by $\sigma\left(T_{w}\right)=T_{\sigma(w)}$ for all $w \in W$ ). Moreover, it permutes the standard basis $\left(T_{w}\right)_{w \in W}$, so it follows from Lemma 2.1 that:

Corollary 2.3. $\quad\left(\operatorname{br}_{G}\left(T_{w}\right)\right)_{w \in W^{G}}$ is an $\mathbb{F}_{p}[\Gamma]$-basis of the $\mathbb{F}_{p}[\Gamma]$-algebra $\operatorname{Br}_{G}(\mathcal{H})$.

Now, let

$$
\operatorname{can}_{G}: \mathcal{H}_{G} \longrightarrow \operatorname{Br}_{G}(\mathcal{H})
$$

be the unique $A$-linear map such that

$$
\operatorname{can}_{G}\left(T_{w}^{G}\right)=\operatorname{br}_{G}\left(T_{w}\right)
$$

for all $w \in W^{G}$. The main result of this subsection is the following:

Proposition 2.4. The map $\operatorname{can}_{G}: \mathcal{H}_{G} \rightarrow \operatorname{Br}_{G}(\mathcal{H})$ is a surjective morphism of A-algebras whose kernel is $p \mathcal{H}_{G}$.

Proof. It follows from Corollary 2.3 that $\operatorname{can}_{G}$ is surjective and that $\operatorname{Ker}\left(\operatorname{can}_{G}\right)=p \mathcal{H}_{G}$. It remains to show that $\operatorname{can}_{G}$ is a morphism of algebras. First, note that if $x, y \in W^{G}$ satisfy $\ell_{G}(x y)=\ell_{G}(x)+\ell_{G}(y)$, then $\ell(x y)=$ $\ell(x)+\ell(y)$ (by Proposition 1.1) and so

$$
\begin{aligned}
\operatorname{can}_{G}\left(T_{x}^{G} T_{y}^{G}\right) & =\operatorname{can}_{G}\left(T_{x y}^{G}\right)=\operatorname{br}_{G}\left(T_{x y}\right) \\
& =\operatorname{br}_{G}\left(T_{x} T_{y}\right)=\operatorname{br}_{G}\left(T_{x}\right) \operatorname{br}_{G}\left(T_{y}\right)=\operatorname{can}_{G}\left(T_{x}^{G}\right) \operatorname{can}_{G}\left(T_{y}^{G}\right) .
\end{aligned}
$$

So it remains to show that, if $\omega$ is a $G$-orbit in $S$ such that $W_{\omega}$ is finite, then

$$
\operatorname{br}_{G}\left(\left(T_{s_{\omega}}-e^{\varphi\left(s_{\omega}\right)}\right)\left(T_{s_{\omega}}+e^{-\varphi\left(s_{\omega}\right)}\right)\right)=0 .
$$

Since $s_{\omega}$ is the longest element of $W_{\omega}$, we have [L, Corollary 12.2]

$$
C_{s_{\omega}}=\sum_{w \in W_{\omega}} e^{\varphi(w)-\varphi\left(s_{\omega}\right)} T_{w}
$$

and [L, Theorem $6.6(\mathrm{~b})]$

$$
\left(T_{s_{\omega}}-e^{\varphi\left(s_{\omega}\right)}\right) C_{s_{\omega}}=0 .
$$

But $\left(W_{\omega}\right)^{G}=\left\{1, s_{\omega}\right\}$. Since $\varphi(w)=\varphi(\sigma(w))$ for all $w \in W_{\omega}$ and all $\sigma \in G$, we have

$$
C_{s_{\omega}} \equiv T_{s_{\omega}}+e^{-\varphi\left(s_{\omega}\right)} \quad \bmod \operatorname{Tr}(\mathcal{H}) .
$$

This completes the proof of (?). 
Corollary 2.5. $\quad \mathbb{F}_{p} \otimes_{\mathbb{Z}} \mathcal{H}_{G} \simeq \operatorname{Br}_{G}(\mathcal{H})$

Corollary 2.6. If $h \in \mathcal{H}_{G}$ and $h^{\prime} \in \mathcal{H}^{G}$ are such that $\operatorname{can}_{G}(h)=$ $\operatorname{br}_{G}\left(h^{\prime}\right)$, then $\tau_{G}(h) \equiv \tau\left(h^{\prime}\right) \bmod p A$.

Proposition 2.7. If $w \in W^{G}$, then $\operatorname{can}_{G}\left(C_{w}^{G}\right)=\operatorname{br}_{G}\left(C_{w}\right)$.

Proof. Let $C=\operatorname{can}_{G}\left(C_{w}^{G}\right)-\operatorname{br}_{G}\left(C_{w}\right)$. Then

$$
\bar{C}=C \text {. }
$$

where ${ }^{-}: \operatorname{Br}_{G}(\mathcal{H}) \rightarrow \operatorname{Br}_{G}(\mathcal{H})$ is defined by $\overline{\operatorname{br}_{G}(h)}=\operatorname{br}_{G}(\bar{h})$ for all $h \in \mathcal{H}^{G}$. Moreover, there exists a family $\left(\alpha_{w}\right)_{w \in W^{G}}$ of elements of $\mathbb{F}_{p} \otimes_{\mathbb{Z}} A_{<0}$ such that

$$
C=\sum_{w \in W^{G}} \alpha_{w} \operatorname{br}_{G}\left(T_{w}\right)
$$

Assume that $C \neq 0$ and let $w$ be maximal (for the Bruhat order) such that $\alpha_{w} \neq 0$. Then

$$
\bar{C}=\bar{\alpha}_{w} \operatorname{br}_{G}\left(T_{w^{-1}}^{-1}\right)+\sum_{\substack{x \in W^{G} \\ x \neq w}} \bar{\alpha}_{x} \operatorname{br}_{G}\left(T_{x^{-1}}^{-1}\right) .
$$

Therefore, the coefficient of $\operatorname{br}_{G}\left(T_{w}\right)$ in $\bar{C}$ is equal to $\bar{\alpha}_{w}$. But $C=\bar{C}$, so $\alpha_{w}=\bar{\alpha}_{w}$. Since $\alpha_{w} \neq 0$ and $\alpha_{w} \in \mathbb{F}_{p} \otimes_{\mathbb{Z}} A_{<0}$, we get a contradiction. So $C=0$, as desired.

Corollary 2.8. If $x, y, z \in W^{G}$, then $h_{x, y, z} \equiv h_{x, y, z}^{G} \bmod p A$ and $\tau\left(C_{z}\right) \equiv \tau_{G}\left(C_{z}^{G}\right) \bmod p A$.

Proof. This follows immediately from Proposition 2.7, from Lemma 2.2 and from Corollary 2.6.

\section{§3. Lusztig's conjectures}

\section{A. Cells}

With $(W, S, \Gamma, \varphi)$ are associated preorder relations $\leqslant_{\mathcal{L}}, \leqslant_{\mathcal{R}}$ and $\leqslant_{\mathcal{L} \mathcal{R}}$ on $W$ as defined in $[\mathrm{L}, \S 8.1]$. The associated equivalence relations are denoted by $\sim_{\mathcal{L}}, \sim_{\mathcal{R}}$ and $\sim_{\mathcal{L} \mathcal{R}}$ respectively. The equivalence classes for the relation $\sim_{\mathcal{L}}$ (respectively $\sim_{\mathcal{R}}$, respectively $\sim_{\mathcal{L} \mathcal{R}}$ ) are called left (respectively right, 
respectively two-sided) cells of $W$ (or for $(W, S, \Gamma, \varphi)$ if it is necessary to emphasize the weight function).

Similarly, with $\left(W^{G}, S_{G}, \Gamma, \varphi_{G}\right)$ are associated preorder relations $\leqslant_{\mathcal{L}}^{G}$, $\leqslant_{\mathcal{R}}^{G}$ and $\leqslant_{\mathcal{L} \mathcal{R}}^{G}$ on $W$. The associated equivalence relations are denoted by $\sim_{\mathcal{L}}^{G}, \sim_{\mathcal{R}}^{G}$ and $\sim_{\mathcal{L} \mathcal{R}}^{G}$ respectively. We shall compare in this section the (left, right or two-sided) cells of $W$ and the ones of $W^{G}$.

\section{B. Boundedness}

Following Lusztig $[\mathrm{L}, \S 13.2]$, we say that $(W, S, \Gamma, \varphi)$ is bounded if there exists $\gamma_{0} \in \Gamma$ such that $\operatorname{deg} \tau\left(T_{x} T_{y} T_{z}\right) \leqslant \gamma_{0}$ for all $x, y$ and $z \in W$. Lusztig has conjectured [L, Conjecture 13.4] that $(W, S, \Gamma, \varphi)$ is always bounded.

Hypothesis. From now on, and until the end of this paper, we assume that $(W, S, \Gamma, \varphi)$ and $\left(W^{G}, S_{G}, \Gamma, \varphi_{G}\right)$ are bounded. Recall that $p$ is a prime number and that $G$ is a finite p-group.

Remark. A finite group is of course bounded. An affine Weyl group is also bounded [L, §13.2].

By [L, Lemma 13.5 (b)], this hypothesis allows us to define Lusztig's function $\mathbf{a}: W \rightarrow \Gamma$ by

$$
\mathbf{a}(z)=\max _{x, y \in W}\left(\operatorname{deg} h_{x, y, z}\right) .
$$

If $x, y, z \in W$, we shall denote by $\gamma_{x, y, z^{-1}}$ the unique element of $\mathbb{Z}$ such that

$$
h_{x, y, z} \equiv \gamma_{x, y, z^{-1}} e^{\mathbf{a}(z)} \bmod \left(\bigoplus_{\gamma<\mathbf{a}(z)} \mathbb{Z} e^{\gamma}\right) .
$$

Similarly, we define a function $\mathbf{a}_{G}: W^{G} \rightarrow \Gamma$ and elements $\gamma_{x, y, z^{-1}}^{G}$ of $\mathbb{Z}$ (for $\left.x, y, z \in W^{G}\right)$.

Let $\mathcal{D}=\{z \in W \mid \mathbf{a}(z)=\Delta(z)\}$. If $I \subseteq S$, we denote by $\mathbf{a}_{I}$ the analogue of the function a but defined for $W_{I}$ instead of $W$ : if $z \in W_{I}$, then

$$
\mathbf{a}_{I}(z)=\max _{x, y \in W_{I}} \operatorname{deg} h_{x, y, z}
$$

Lusztig's Conjectures FOR $(W, S, \Gamma, \varphi)$. With the above notation, we have:

$\boldsymbol{P}_{\mathbf{1}}$. If $z \in W$, then $\mathbf{a}(z) \leqslant \Delta(z)$.

$\boldsymbol{P}_{\mathbf{2}}$. If $d \in \mathcal{D}$ and if $x, y \in W$ satisfy $\gamma_{x, y, d} \neq 0$, then $x=y^{-1}$. 
$\boldsymbol{P}_{\mathbf{3}}$. If $y \in W$, then there exists a unique $d \in \mathcal{D}$ such that $\gamma_{y^{-1}, y, d} \neq 0$.

$\boldsymbol{P}_{\mathbf{4}}$. If $z^{\prime} \leqslant_{\mathcal{L} \mathcal{R}} z$, then $\mathbf{a}(z) \leqslant \mathbf{a}\left(z^{\prime}\right)$. Therefore, if $z \sim_{\mathcal{L} \mathcal{R}} z^{\prime}$, then $\mathbf{a}(z)=$ $\mathbf{a}\left(z^{\prime}\right)$.

$\boldsymbol{P}_{\mathbf{5}}$. If $d \in \mathcal{D}$ and $y \in W$ satisfy $\gamma_{y^{-1}, y, d} \neq 0$, then $\gamma_{y^{-1}, y, d}=n_{d}= \pm 1$.

$\boldsymbol{P}_{6}$. If $d \in \mathcal{D}$, then $d^{2}=1$.

$\boldsymbol{P}_{\mathbf{7}}$. If $x, y, z \in W$, then $\gamma_{x, y, z}=\gamma_{y, z, x}$.

$\boldsymbol{P}_{8}$. If $x, y, z \in W$ satisfy $\gamma_{x, y, z} \neq 0$, then $x \sim_{\mathcal{L}} y^{-1}, y \sim_{\mathcal{L}} z^{-1}$ and $z \sim \mathcal{L} x^{-1}$.

$\boldsymbol{P}_{\mathbf{9}}$. If $z^{\prime} \leqslant_{\mathcal{L}} z$ and $\mathbf{a}\left(z^{\prime}\right)=\mathbf{a}(z)$, then $z^{\prime} \sim_{\mathcal{L}} z$.

$\boldsymbol{P}_{\mathbf{1 0}}$. If $z^{\prime} \leqslant_{\mathcal{R}} z$ and $\mathbf{a}\left(z^{\prime}\right)=\mathbf{a}(z)$, then $z^{\prime} \sim_{\mathcal{R}} z$.

$\boldsymbol{P}_{11}$. If $z^{\prime} \leqslant_{\mathcal{L R}} z$ and $\mathbf{a}\left(z^{\prime}\right)=\mathbf{a}(z)$, then $z^{\prime} \sim_{\mathcal{L R}} z$.

$\boldsymbol{P}_{\mathbf{1 2}}$. If $I \subset S$ and $z \in W_{I}$, then $\mathbf{a}_{I}(z)=\mathbf{a}(z)$.

$\boldsymbol{P}_{\mathbf{1 3}}$. Every left cell $\mathcal{C}$ of $W$ contains a unique element $d \in \mathcal{D}$. If $y \in \mathcal{C}$, then $\gamma_{y^{-1}, y, d} \neq 0$.

$\boldsymbol{P}_{14}$. If $z \in W$, then $z \sim_{\mathcal{L R}} z^{-1}$.

$\boldsymbol{P}_{\mathbf{1 5}}$. If $x, x^{\prime}, y, w \in W$ are such that $\mathbf{a}(y)=\mathbf{a}(w)$, then

$$
\sum_{y^{\prime} \in W} h_{w, x^{\prime}, y^{\prime}} \otimes_{\mathbb{Z}} h_{x, y^{\prime}, y}=\sum_{y^{\prime} \in W} h_{y^{\prime}, x^{\prime}, y} \otimes_{\mathbb{Z}} h_{x, w, y^{\prime}}
$$

in $A \otimes_{\mathbb{Z}} A$.

Let us recall the following result:

Lemma 3.1. Assume that Lusztig's Conjectures $\left(P_{1}\right),\left(P_{2}\right),\left(P_{3}\right)$ and $\left(P_{4}\right)$ hold for $(W, S, \Gamma, \varphi)$. Then:

(a) Lusztig's Conjectures $\left(P_{5}\right),\left(P_{6}\right),\left(P_{7}\right)$ and $\left(P_{8}\right)$ hold for $(W, S, \Gamma, \varphi)$.

(b) If $d \in \mathcal{D}$, then $\gamma_{d, d, d}=n_{d}= \pm 1$.

(c) If $x \in W$ and if $d \in \mathcal{D}$ is the unique element of $W$ such that $\gamma_{x^{-1}, x, d} \neq$ 0 , then $\gamma_{x, d, x^{-1}}= \pm 1$.

Proof. (a) is proved in [L, Chapter 14].

(b) By $\left(P_{6}\right)$, we get that $d^{2}=1$. By $\left(P_{3}\right)$, there exists a unique $e \in \mathcal{D}$ such that $\gamma_{d, d, e} \neq 0$. By $\left(P_{5}\right)$, this implies that $\gamma_{d, d, e}=n_{e}= \pm 1$. By $\left(P_{7}\right)$, this implies that $\gamma_{e, d, d}= \pm 1$. By $\left(P_{2}\right)$, we get that $e=d^{-1}=d$.

(c) If $x \in W$ and if $d \in \mathcal{D}$ is the unique element of $W$ such that $\gamma_{x^{-1}, x, d} \neq 0$, then $\gamma_{x, d, x^{-1}}=\gamma_{x^{-1}, x, d}= \pm 1$ by $\left(P_{7}\right)$ and $\left(P_{5}\right)$. 
We can now state the main result of this paper (from which the Theorem $\mathrm{A}$ in the introduction follows easily by an induction argument on the order of $G)$ :

TheOREM 3.2. Recall that $G$ is a finite p-group. Assume that Lusztig's conjectures $\left(P_{1}\right),\left(P_{2}\right),\left(P_{3}\right)$ and $\left(P_{4}\right)$ hold for both $(W, S, \Gamma, \varphi)$ and $\left(W^{G}, S_{G}\right.$, $\left.\Gamma, \varphi_{G}\right)$. Let $x$ and $y$ be two elements of $W^{G}$. Then:

(a) $\mathbf{a}_{G}(x)=\mathbf{a}(x)$.

(b) $\mathcal{D}_{G}=\mathcal{D} \cap W^{G}\left(=\mathcal{D}^{G}\right)$.

(c) Assume moreover that Lusztig's Conjecture $\left(P_{13}\right)$ holds for both $(W, S$, $\Gamma, \varphi)$ and $\left(W^{G}, S_{G}, \Gamma, \varphi_{G}\right)$. Then $x \sim_{\mathcal{L}}^{G} y$ (respectively $\left.x \sim_{\mathcal{R}}^{G} y\right)$ if and only if $x \sim_{\mathcal{L}} y$ (respectively $\left.x \sim_{\mathcal{R}} y\right)$.

$(d)$ Assume moreover that Lusztig's Conjectures $\left(P_{9}\right)$ and $\left(P_{13}\right)$ hold for both $(W, S, \Gamma, \varphi)$ and $\left(W^{G}, S_{G}, \Gamma, \varphi_{G}\right)$. Then $x \sim_{\mathcal{L} \mathcal{R}}^{G} y$ if and only if $x \sim_{\mathcal{L R}} y$.

Proof. (a) By Corollary 2.8, we have, for all $x, y, z \in W^{G}$ :

(1) If $\gamma_{x, y, z^{-1}} \not \equiv 0 \bmod p$, then $\mathbf{a}(z) \leqslant \mathbf{a}_{G}(z)$.

(2) If $\gamma_{x, y, z^{-1}}^{G} \not \equiv 0 \bmod p$, then $\mathbf{a}_{G}(z) \leqslant \mathbf{a}(z)$.

Now let $z \in W^{G}$. By $\left(P_{3}\right)$, there exists a unique $d \in \mathcal{D}$ such that $\gamma_{z^{-1}, z, d} \neq 0$. From the uniqueness, we get that $d \in \mathcal{D}^{G} \subseteq W^{G}$. By Lemma 3.1 (c), we get that $\gamma_{z, d, z^{-1}}= \pm 1$. So $\mathbf{a}(z) \leqslant \mathbf{a}_{G}(z)$ by $(1)$.

The same argument shows that there exists $d \in \mathcal{D}_{G}$ such that $\gamma_{z, d, z^{-1}}^{G}=$ \pm 1 , so (2) can be applied to get that $\mathbf{a}_{G}(z) \leqslant \mathbf{a}(z)$. The proof of (a) is complete.

Before going further, let us state the following consequence of (a):

Corollary 3.3. If $x, y, z \in W^{G}$, then $\gamma_{x, y, z} \equiv \gamma_{x, y, z}^{G} \bmod p$.

Proof. This follows easily from Theorem 3.2 (a) and Corollary 2.8.

(b) Let $d \in \mathcal{D}^{G}$. By Lemma 3.1 (b), we have $n_{d}= \pm 1$. Moreover, by Corollary 2.8, we have

$$
\tau\left(C_{d}\right) \equiv \tau_{G}\left(C_{d}^{G}\right) \quad \bmod p A
$$


This shows that the coefficient of $e^{-\Delta(d)}$ in $\tau_{G}\left(C_{d}^{G}\right)$ is non-zero. So $\Delta_{G}(d) \leqslant$ $\Delta(d)$. But, by $\left(P_{1}\right)$,

$$
\mathbf{a}_{G}(d) \leqslant \Delta_{G}(d) \leqslant \Delta(d)=\mathbf{a}(d)
$$

So $\mathbf{a}_{G}(d)=\Delta_{G}(d)=\Delta(d)=\mathbf{a}(d)$ by (a). In particular, $d \in \mathcal{D}_{G}$.

The same argument shows that, if $d \in \mathcal{D}_{G}$, then $\Delta(d) \leqslant \Delta_{G}(d)$ and again we get similarly that $d \in \mathcal{D}$. The proof of (b) is complete.

(c) Let $d$ (respectively $e$ ) be the unique element of $\mathcal{D}$ such that $\gamma_{x^{-1}, x, d}=$ \pm 1 (respectively $\gamma_{y^{-1}, y, e}= \pm 1$ ). By uniqueness, we have $d, e \in \mathcal{D}^{G}=\mathcal{D}_{G}$. By Corollary 3.3, we also get $\gamma_{x^{-1}, x, d}^{G} \neq 0$ and $\gamma_{y^{-1}, y, e}^{G} \neq 0$. Therefore, by $\left(P_{8}\right)$, we have

$$
x \sim_{\mathcal{L}} d, \quad x \sim_{\mathcal{L}}^{G} d, \quad y \sim_{\mathcal{L}} e \text { and } y \sim_{\mathcal{L}}^{G} e .
$$

But, by $\left(P_{13}\right)$, we have $x \sim_{\mathcal{L}} y$ (respectively $x \sim_{\mathcal{L}}^{G} y$ ) if and only if $d=e$. This proves $(\mathrm{c})$.

(d) Recall that $\left(P_{9}\right)$ implies $\left(P_{10}\right)$. Moreover, it follows easily from $\left(P_{4}\right),\left(P_{9}\right)$ and $\left(P_{10}\right)$ that $\sim_{\mathcal{L} \mathcal{R}}$ (respectively $\left.\sim_{\mathcal{L} \mathcal{R}}^{G}\right)$ is the equivalence relation generated by $\sim_{\mathcal{L}}$ and $\sim_{\mathcal{R}}$ (respectively $\sim_{\mathcal{L}}^{G}$ and $\sim_{\mathcal{R}}^{G}$ ). So (d) follows from (c).

\section{C. Asymptotic algebra}

Let $J$ (respectively $J_{G}$ ) be the free abelian group with basis $\left(t_{w}\right)_{w \in W}$ (respectively $\left.\left(t_{w}^{G}\right)_{w \in W}\right)$.

Hypothesis. In this subsection, and only in this subsection, we assume moreover that Lusztig's Conjectures $\left(P_{1}\right),\left(P_{2}\right), \ldots,\left(P_{15}\right)$ hold for $(W, S, \Gamma, \varphi)$ and $\left(W^{G}, S_{G}, \Gamma, \varphi_{G}\right)$.

By $[\mathrm{L}, \S 18.3], J$ (respectively $J_{G}$ ) can be endowed with a structure of associative ring, the multiplication being defined by $t_{x} t_{y}=\sum_{z \in W} \gamma_{x, y, z^{-1}} t_{z}$ (respectively $t_{x}^{G} t_{y}^{G}=\sum_{z \in W^{G}} \gamma_{x, y, z^{-1}}^{G} t_{z}^{G}$ ). Then it follows immediately from Corollary 3.3 and from Lemma 2.2 that:

TheOrem 3.4. Assume that $G$ is a finite p-group and that Lusztig's Conjectures $\left(P_{1}\right),\left(P_{2}\right), \ldots,\left(P_{15}\right)$ hold for $(W, S, \Gamma, \varphi)$ and $\left(W^{G}, S_{G}, \Gamma, \varphi_{G}\right)$. Then

$$
\mathbb{F}_{p} \otimes_{\mathbb{Z}} J_{G} \simeq \operatorname{Br}_{G}(J)
$$




\section{§4. Open questions}

The results of this paper should be compared with [L, Chapter 14], where the quasi-split case is considered: more particularly, see [L, Lemmas 16.5, 16.6 and 16.14]. This leads to the following questions:

- Does Theorem A (d) hold if $G$ is not solvable? It is probably the case, but a proof should rely on completely different arguments. For the statements (a), (b) and (c), see the remark after Theorem A in the introduction.

- Let $z \in W^{G}$. Is it true that $\Delta(z) \leqslant \Delta_{G}(z)$ ? See [L, Lemma 16.5] for the quasi-split case.

- Let $x, y \in W^{G}$ be such that $x \leqslant_{\mathcal{L}}^{G} y$. Is it true that $x \leqslant_{\mathcal{L}} y$ ? See $[\mathrm{L}$, 16.13 (a)] for the quasi-split case.

\section{REFERENCES}

[G] M. Geck, On the induction of Kazhdan-Lusztig cells, Bull. London Math. Soc., 35 (2003), 608-614.

[H] J.-Y. Hée, Systèmes de racines sur un anneau commutatif totalement ordonné, Geometriae Dedicata, 37 (1991), 65-102.

[L] G. Lusztig, Hecke algebras with unequal parameters, CRM Monograph Series 18, American Mathematical Society, Providence, RI, 2003, 136 pp.

[T] J. Thévenaz, G-Algebras and Modular Representation Theory, Clarendon Press, Oxford, 1995.

Labo. de Math. de Besançon (CNRS: UMR 6623)

Université de Franche-Comté

16 Route de Gray

25030 Besançon Cedex

France

cedric.bonnafe@univ-fcomte.fr 